\section{Assessment of left ventricular functions by tissue Doppler imaging in patients with ankylosing spondylitis}

\author{
Osman Kuloglu, Nihal Akar Bayram, \\ Sukran Erten, Tahir Durmaz, Telat Keles, \\ Cenk Sari, Murat Akcay, Engin Bozkurt
}

Ataturk Education and Research Hospital, Ankara, Turkey

\section{Abstract}

Ankylosing spondylitis (ASp) is a chronic, inflammatory and systemic disease affecting pericardium, myocardium and the conduction system of the heart. In this study, we aimed to analyse left ventricular systolic and diastolic functions using tissue Doppler imaging (TDI). 30 patients with ASp and 30 healthy volunteers having the similar demographic characteristics were included. Left ventricular systolic and diastolic functions were assessed by using two dimensional (2D) echocardiography, M-mode, pulsed-wave (PW) and tissue Doppler echocardiography. The peak systolic velocity (Sm), early diastolic myocardial peak velocity (m), late diastolic myocardial peak velocity (Am), isovolumic acceleration (IVA), myocardial precontraction time (PCTm), myocardial contraction time $(\mathrm{CTm})$, myocardial relaxation time (RTm), and myocardial performance index (MPI) were measured at septal and lateral mitral annulus. In conventional echocardiography, end-diastolic interventricular septum and posterior wall diameters were higher in patients with ASp than the control group. The ratio of E/A was significantly lower and deceleration time was significantly prolonged in patients with ASp, but mitral $\mathrm{E}$ and A velocities, isovolumic relaxation time and MPI were similar in patient and control group ( $\mathrm{P}>0.05)$. Left ventricular lateral and septal wall tissue Doppler echocardiography showed that Em, Em/Am ratio and CTm were significantly lower, IVRTm was longer and MPI was higher in patients with ASp. No significant differences were detected between the groups for IVA, Sm, Am, PCTm, PCTm/CTm ratio ( $\mathrm{P}>0.05)$. We have demonstrated that in patients with $\mathrm{ASp}$, diastolic functions were impaired but systolic functions were preserved by using TDI.

\section{Introduction}

Ankylosing spondilitis (ASp) is a chronic, inflammatory and systemic disease affecting the axial system. Besides the axial skeleton, peripheral joints, eye, muscles, pulmonary, gastrointestinal, genitourinary, and cardiovascular systems can be affected. In ASp, ascending aorta, aorta and mitral valve, myocardium, pericardium, and cardiac conducting systems can be affected. ${ }^{1-5}$ Although cardiovascular disease has important clinical and prognostic signs in ASp, it is usually undiagnosed. Conventional clinical, radiologic and serologic findings of the disease are unable to diagnose cardiovascular disease.1,6 The most frequent cause of death in ASp is heart diseases. The prevalence of heart failure is higher in ASp patients than the normal population., 1,5,7,

HLA B27 positivity is an important risk factor for development of cardiac symptoms. ${ }^{9}$ Histopathologically, adventitial scarring, intimal proliferation and lymphocyte and plasma cell infiltration of vaso vasorum are prominant features of cardiovascular system. ${ }^{10}$ Fibrous tissue accumulates on membraneous part of interventricular septum below the aortic valve. Aortic insufficiency or ascending aorta dilatation can occur due to fibrotic shortening of aortic valve leaflets. Mitral insufficiency occurs due to dispension of fibrosis to anterior mitral leaflet. Fibrosis of interventricular system can lead to conduction system abnormalities. Overproduction of connective tissue affects myocardial functions.

Although there were several studies evaluating left ventricular systolic and diastolic functions by conventional echocardiography, limited number of studies were used tissue Doppler imaging (TDI) in AS.4,8,11,12 Therefore, the aim of the present study was to analyse systolic and diastolic functions of the left ventricle by using tissue Doppler echocardiography and myocardial performance index (MPI) together with conventional methods.

\section{Materials and Methods}

This study included 30 patients with ASp (18 male, 12 female, mean age $37.2 \pm 10.23$ years) who were diagnosed according to modified New York Criteria and 30 healthy volunteers (16 male, 14 female, mean age $33.2 \pm 8.12$ years). The study was approved by the Ethics Committee Ankara Atatürk Education and Research Hospital.

Exclusion criteria were patients who were older than 60 or younger than 18 years, or who have heart diseases, hypertension, diabetes mellitus, pulmonary, neoplastic or other chronic diseases.

\section{Echocardiography}

All echocardiographic images were obtained with a scanner (Vingmed System 7; Vivid 7 Pro; Horten, Norway) using a 2,5 to $3,5 \mathrm{MHz}$ probe. Echocardiographic measurements were
Correspondence: Şükran Erten, Department of Rheumatology, Ataturk Education and Research Hospital, Bilkent, Ankara, Turkey.

Tel. +90.312.291.2525 - Fax: +90.312.291.2705.

E-mail: sukranerten@yahoo.com

Key words: ankylosing spondylitis, left ventricular systolic function, left ventricular diastolic function, tissue Doppler echocardiography.

Conflict of interests: the authors report no conflict of interests.

Received for publication: 3 August 2011.

Revision received: 23 March 2012.

Accepted for publication: 30 March 2012.

This work is licensed under a Creative Commons Attribution NonCommercial 3.0 License (CC BYNC 3.0).

(C) Copyright O. Kuloglu et al., 2012

Licensee PAGEPress, Italy

Rheumatology Reports 2012; 4:e6

doi:10.4081/rr.2012.e6

taken with patients in the left lateral decubitis position according to the recommendations of American Society of Echocardiography. ${ }^{13}$ All measurements were the average of values obtained from 3 to 5 beats. The echocardiographic study was performed by an experienced operator who was blinded to the clinical status of the subject.

Left atrial diameter (LA; mm), left ventricle end diastolic diameter (LVEDD; mm), left ventricle end systolic diameter (LVESD; mm), interventricular septum diameter (IVSd; mm) at end diastole, posterior wall diameter (PWd; $\mathrm{mm}$ ) at end diastole were obtained from the Mmode echocardiographic tracing under the guide of two-dimensional imaging. Left ventricular ejection fraction was calculated with Simpson's method as (diastolic volume-systolic volume)/(diastolic volume).

The transmitral diastolic flow velocities were measured in the apical four-chamber view by using pulsed Doppler echocardiography with the sample volume sited at the tip of mitral leaflet. Mitral early diastolic flow (E) velocity and late diastolic flow (A) velocity, ET (ejection time), IVRT (isovolumetric relaxation time), and IVCT (isovolumetric contraction time) were measured from an apical fourchamber view, and myocardial performance index (Tei index) was calculated. Time intervals used to calculate the Tei index were measured using pulsed-wave Doppler velocity spectra of ventricular inflow and outflow Tei index; $(\mathrm{a}-\mathrm{b}) / \mathrm{b}$ means (IVRT+IVCT)/ET. The interval a is equal to the sum of IVCT, IVRT, and ET. The interval b is the left ventricle outflow ET.

Tissue Doppler measurements were performed in the apical four-chamber view, and 3 $\mathrm{mm}$ a pulsed Doppler sample volume was placed 
at the level of septal mitral annulus and lateral mitral annulus. The peak systolic velocity (S'm; centimeters per second), early diastolic myocardial peak velocity (E'm; centimeters per second), late diastolic myocardial peak velocity (A'm; centimeters per second), isovolumetric acceleration (IVA; meter per second squared; precedes S'm and was calculated by dividing myocardial peak velocity during isovolumic contraction by the time interval from the onset of this wave to the time at peak velocity), myocardial pre-contraction time (PCT'm; milliseconds; time interval between the onset of electrocardiograms QRS and onset of S'm), myocardial contraction time (CT'm; milliseconds; between onset and cessation of the $S^{\prime} m$ ) and myocardial relaxation time (RT'm; milliseconds; from the end of S'm to the onset of E'm), E to E'm ratio (was the ratio between the $\mathrm{E}$ transmitral flow velocity and the mean of lateral and septal walls E'm velocity) were measured.

S'm velocity, PCT'm, PCT'm/CT'm, IVA, PEP, PEP/EP were used as LV systolic function parameters. E velocity, A velocity, DT, IVRT, E to A ratio, E'm velocity, A'm velocity, E to E'm ratio, RT'm were determined as LV diastolic function parameters. All measurements were averaged for three consecutive cycles.

\section{Statistics}

The statistical package for social sciences (SPSS) version 11.5 was used for the statistical analysis. Data were expressed as arithmetic mean \pm SD or median (min-max) for numerical variables and counts and percentage for categoric variables. Differences between patient groups and controls were determined by using Mann Whitney U test or Student's $t$-test. Categoric variables were compared using Pearson Chi-Square tests. $P$ values $<0.05$ were considered as significant.

\section{Results}

The characteristics of the subjects were presented in Table 1. There was no statistically significant difference between the patients with ASp and controls with respect to age, sex, body mass index, systolic blood pressure, diastolic blood pressure, heart rate, and cigarettte smoking $(\mathrm{P}<0.05)$.

The results of conventional echocardiographic examination were displayed in Table 2 . LA, LVEDD, LVESD and EF were not significantly different between the groups. IVSd $(10.4 \pm 1.5 \mathrm{~mm}$ vs. $9.6 \pm 1.2 \mathrm{~mm} ; \mathrm{P}=0.04)$ and PWd $(9.9 \pm 0.9 \mathrm{~mm}$ vs. $9.3 \pm 1.3 \mathrm{~mm} ; \mathrm{P}=0.03)$ were higher in patients with ASp than controls. The ratio of $\mathrm{E} / \mathrm{A}$ was significantly lower $(1.2 \pm 0.24$ vs. $1.4 \pm 0.32 ; \mathrm{P}=0.02)$ and, DT $(196.6 \pm 32.6 \mathrm{~ms}$ vs. $169.0 \pm 37.4 \mathrm{~ms} ; \mathrm{P}=0.003)$ was significantly prolonged in patients with
ASp, but mitral E and A velocities, PEP, EP, PEP to EP ratio, IVRT and MPI were similar in patients and control group ( $\mathrm{P}>0.05)$.

Lateral wall TDI findings were summarized in Table 3. Em, Em/Am ratio and CTm were significantly lower, IVRTm was longer and MPI was higher in patients with ASp than controls. No significant differences were detected between the groups for IVA, Sm, Am, PCTm, $\mathrm{PCTm} / \mathrm{CTm}$ ratio $(\mathrm{P}>0.05)$.

Septal wall TDI parameters were compared between patients with ASp and controls in Table 4. In patients with ASp Em, Em/Am ratio and CTm were significantly lower, IVRTm was longer and MPI was higher than the control group. There were no significant differences between IVA, Sm, Am, PCTm, PCTm/CTm ratio among the groups.

MPI value of the lateral wall and septum which was calculated by TDI was $53 \%$ in ASp and $20 \%$ in control group which was statistically significant $(\mathrm{P}=0.01)$.

\section{Discussion}

In the present study, it was shown that left ventricle systolic functions were preserved, but diastolic functions were impaired in patients

Table 1. Clinical characteristics of patients with ankylosing spondylitis and the control group.

\begin{tabular}{lccc} 
& Patients $(\mathrm{n}=30)$ & Control group $(\mathrm{n}=30)$ & $\mathrm{P}$ \\
Age (year) & $37.2 \pm 10.23$ & $33.2 \pm 8.12$ & NS \\
Sex $(\mathrm{M} / \mathrm{F})$ & $18 / 12$ & $16 / 14$ & NS \\
\hline Body mass index $\left(\mathrm{kg} / \mathrm{m}^{2}\right)$ & $26.0 \pm 2.69$ & $25.2 \pm 3.05$ & NS \\
Systolic blood pressure $(\mathrm{mmHg})$ & $118.1 \pm 8.31$ & $121.5 \pm 7.53$ & NS \\
\hline Diastolic blood pressure $(\mathrm{mmHg})$ & $71.3 \pm 7.86$ & $74.0 \pm 7.80$ & NS \\
pulse (beats/min) & $73.7 \pm 8.70$ & $70.1 \pm 5.69$ & NS \\
\hline Cigarette smoking (\%) & $11(\% 36.7)$ & $11(\% 36.7)$ & NS \\
Heart rate (beats/min) & $70.1 \pm 3.4$ & $68.2 \pm 6.6$ & NS \\
\hline Fasting blood glucose (mg/dl) & $118.6 \pm 34.6$ & $110.7 \pm 20.9$ & NS \\
Cholesterol (mg/dl) & $195.6 \pm 45.6$ & $191.3 \pm 28.3$ & NS \\
\hline LDL-cholesterol (mg/dl) & $126.4 \pm 30.8$ & $114.8 \pm 24.1$ & NS \\
HDL-cholesterol (mg/dl) & $38.7 \pm 5.6$ & $41.6 \pm 6.7$ & NS \\
\hline Triglyceride (mg/dl) & $165.6 \pm 68.4$ & $151.4 \pm 20.1$ & NS \\
ESR (mm/h) & $23.8 \pm 16.7$ & $4.5 \pm 4.1$ & $<0.001$ \\
\hline
\end{tabular}

ESR, erythrocyte sedimentation rate; NS, non significant.

Table 2. Conventional echocardiographic results in study groups.

\begin{tabular}{lccc} 
& Patients $(\mathrm{n}=30)$ & Control group $(\mathrm{n}=30)$ & $\mathrm{P}$ \\
LA, mm & $46.6 \pm 3.9$ & $45.5 \pm 2.4$ & $\mathrm{NS}$ \\
LVEDD, $\mathrm{mm}$ & $47.0 \pm 3.4$ & $48.3 \pm 3.0$ & $\mathrm{NS}$ \\
\hline LVESD, mm & $30.0 \pm 3.1$ & $29.6 \pm 2.8$ & $\mathrm{NS}$ \\
EF $(\%)$ & $65.6 \pm 4.7$ & $67.5 \pm 3.7$ & $\mathrm{NS}$ \\
\hline IVSd, mm & $10.4 \pm 1.5$ & $9.6 \pm 1.2$ & 0.04 \\
PWd, mm & $9.9 \pm 0.9$ & $9.3 \pm 1.3$ & 0.03 \\
\hline E wave, $\mathrm{m} / \mathrm{s}$ & $0.83 \pm 0.14$ & $0.89 \pm 0.18$ & NS \\
A wave, $\mathrm{m} / \mathrm{s}$ & $0.69 \pm 0.13$ & $0.63 \pm 0.10$ & $\mathrm{NS}$ \\
\hline E to A ratio & $1.23 \pm 0.24$ & $1.41 \pm 0.32$ & 0.02 \\
DT, ms & $196.6 \pm 32.6$ & $169.0 \pm 37.4$ & 0.003 \\
\hline IVRT, ms & $93.1 \pm 9.7$ & $97.7 \pm 19.3$ & NS \\
PEP, ms & $82.2 \pm 8.5$ & $79.9 \pm 10.2$ & NS \\
\hline EP, ms & $265.6 \pm 26.5$ & $267.2 \pm 28.7$ & NS \\
PEP to EP ratio & $0.31 \pm 0.04$ & $0.30 \pm 0.06$ & NS \\
\hline MPI & $0.66 \pm 0.09$ & $0.67 \pm 0.16$ & NS \\
\hline
\end{tabular}

A, mitral late diastolic velocity; DT, deceleration time of E; EP, ejection time; EF, ejection fraction; E, mitral early diastolic velocity; IVSd, interventricular septum diameter at end diastole; LVEDD, Left ventricle end diastolic diameter; LA, left atrium; LVESD, left ventricle end systolic diameter; LVM, left ventricle mass; LVMI, left ventricle mass index; MPI, myocardial performance index; PWd, posterior wall diameter at end diastole; PEP, preejection period; RWT, relative wall thickness; IVRT, isovolumetric relaxation time, ms: millisecond; NS, not significant. 
with ASp. Contradictory results were obtained from the studies evaluating left ventricle functions in patients with AS. ${ }^{8,11,14}$ Methodological differences and limitations of conventional echocardiographic methods to diagnose left ventricular function may cause these discrepancy. Brewerton et al. ${ }^{4}$ have evaluated LV function by two dimensional and M-mode echocardiography in ASp patients. They found longer IVRT and increased mitral valve opening in miliseconds and increased interval from aortic valve closure to mitral valve opening in miliseconds.

In a study performed by Sun et al. ${ }^{14} \mathrm{E}$ and E/A ratio were lower for both mitral and tricuspid valves and diastolic influx time was shorter in ASp patients. These findings demonstrated that diastolic dysfunction was present in ASp patients. In the two other studies, frequency of LV diastolic dysfunction in ASp patients was found to be $20 \%$ and $26 \%$ respectively. ${ }^{11,12}$

The present study has showed that mitral E and A velocities, PEP, EP, PEP/EP, IVRT and MPI were not different between two groups. E/A ratio was decreased and DT was longer signif- icantly in ASp patients than controls, and diastolic dysfunction was found as $26 \%$ similar to other studies using to conventional echocardiography. Left ventricle dysfunction in ASp has been attributed to several factors. ${ }^{4,15}$ These include diffuse increase in myocardial interstitial connective tissue, myocarditis, amyloidosis, aortic insufficieny, conduction disturbances, cardiomyopathy, pericarditis and mitral valve diseases..$^{15-17}$

TDI allows quantitative measurements of myocardial contraction and relaxation velocities of a selected myocardial segment. TDI allows providing velocities of normal and pathologic myocardial structures during the cardiac cycle. Evaluation of myocardial wall velocities with respect to timing and amplitude has been suggested for quantification of global and regional systolic and diastolic functions. Preload and afterload may affect diastolic filling patterns measured by conventional echocardiography. On the contrary, analysis of signals with high amplitude and low frequency and wall motion velocities were measured by

Table 3. Tissue Doppler imaging measurements of lateral wall in groups.

\begin{tabular}{lccc} 
& Patients $(\mathrm{n}=30)$ & Control group $(\mathbf{n}=30)$ & $\mathrm{P}$ \\
$\mathrm{Sm}, \mathrm{cm} / \mathrm{s}$ & $11.0 \pm 2.7$ & $11.0 \pm 2.5$ & $\mathrm{NS}$ \\
$\mathrm{IVA}, \mathrm{m} / \mathrm{s}^{2}$ & $3.6 \pm 0.7$ & $3.6 \pm 0.8$ & $\mathrm{NS}$ \\
\hline $\mathrm{Em}, \mathrm{cm} / \mathrm{s}$ & $12.1 \pm 2.4$ & $13.9 \pm 3.0$ & 0.01 \\
$\mathrm{Am}, \mathrm{cm} / \mathrm{s}$ & $9.0 \pm 2.8$ & $8.4 \pm 3.0$ & $\mathrm{NS}$ \\
\hline $\mathrm{Em} / \mathrm{Am}$ ratio & $1.4 \pm 0.4$ & $1.8 \pm 0.7$ & 0.03 \\
$\mathrm{PCTm}, \mathrm{ms}$ & $75.2 \pm 11.0$ & $73.7 \pm 8.0$ & $\mathrm{NS}$ \\
\hline CTm, msec & $264.9 \pm 19.1$ & $278.3 \pm 23.3$ & 0.02 \\
PCTm/CTm ratio & $0.28 \pm 0.04$ & $0.26 \pm 0.04$ & $\mathrm{NS}$ \\
\hline IVRTm, ms & $88.1 \pm 9.0$ & $82.3 \pm 9.6$ & 0.02 \\
MPI & $0.80 \pm 0.07$ & $0.56 \pm 0.07$ & 0.03 \\
\hline
\end{tabular}

Am, late diastolic myocardial peak velocity; CTm, myocardial contraction; Em, early diastolic myocardial peak velocity; IVA, isovolumic acceleration time; MPI, Myocardial performance index; PCTm, myocardial precontraction time; Sm, peak systolic myocardial velocity; NS, Non significant.

Table 4. Tissue Doppler imaging measurements of septal wall in groups.

\begin{tabular}{lccc} 
& Patients $(\mathrm{n}=30)$ & Control group $(\mathrm{n}=30)$ & $\mathrm{P}$ \\
$\mathrm{Sm}, \mathrm{cm} / \mathrm{s}$ & $9.3 \pm 1.5$ & $10.1 \pm 2.0$ & $\mathrm{NS}$ \\
$\mathrm{IVA}, \mathrm{m} / \mathrm{s}^{2}$ & $3.7 \pm 1.0$ & $3.4 \pm 0.9$ & $\mathrm{NS}$ \\
\hline $\mathrm{Em}, \mathrm{cm} / \mathrm{s}$ & $10.1 \pm 2.3$ & $11.6 \pm 2.1$ & 0.007 \\
$\mathrm{Am}, \mathrm{cm} / \mathrm{s}$ & $8.5 \pm 1.9$ & $8.1 \pm 2.0$ & $\mathrm{NS}$ \\
\hline Em/Am ratio & $1.2 \pm 0.4$ & $1.5 \pm 0.4$ & 0.03 \\
PCTm, ms & $78.6 \pm 10.2$ & $75.2 \pm 8.3$ & $\mathrm{NS}$ \\
\hline CTm, msec & $265.9 \pm 21.6$ & $278.7 \pm 23.1$ & 0.03 \\
PCTm/CTm ratio & $0.29 \pm 0.05$ & $0.27 \pm 0.04$ & $\mathrm{NS}$ \\
\hline IVRTm, ms & $87.3 \pm 11.1$ & $79.8 \pm 11.4$ & 0.01 \\
MPI & $0.62 \pm 0.07$ & $0.56 \pm 0.09$ & 0.004 \\
\hline
\end{tabular}

Am, late diastolic myocardial peak velocity; CTm, myocardial contraction; Em, early diastolic myocardial peak velocity; IVA, isovolumic acceleration time; MPI, Myocardial performance index; PCTm, myocardial precontraction time; Sm, peak systolic myocardial velocity; NS, Non significant.

TDI relatively irrespective of volume. Despite a preserved global function, TDI may represent an early stage of myocardial abnormality. ${ }^{18-20}$

There are some studies using TDI in ASp patients. Okan and colleagues evaluated LV diastolic functions by using standart Doppler echocardiography (SDE) and TDI in patients with ASp..$^{21}$ They founded no significant difference between ASp patients and control group with regard to the measurements for LV diastolic dysfunction by using SDE. On the other hand, by using TDI method, they detected isolated LV diastolic dysfunction in $47 \%$ of ASp patients. They also demostrated prolonged LV MPI in patients with ASp by both standart and tissue Doppler echocardiography. In a study performed by Calıskan et al., ${ }^{22} \mathrm{LV}$ diastolic functions were evaluated in ASp patients. Mitral E and A wave velocities and $\mathrm{E} / \mathrm{A}$ ratio, lateral $\mathrm{Em}, \mathrm{Am}$ and Em/Am ratio were not significantly different between groups. However, mitral A wave deceleration time and IVRT were longer and lateral Am was higher significantly in patients with ASp. In these studies, diastolic functions of the ASp patients were analysed, but in the present study, besides the left ventricle diastolic functions, systolic functions were also evaluated.

\section{Conclusions}

In the present study, lateral and septal wall $\mathrm{Em}, \mathrm{Em} / \mathrm{Am}$ ratio and CTm were lower, IVRTm and MPI was higher significantly in AS patients than the control group with TDI. By using SDE, no significant difference was detected between groups with regard to the measurements made to evaluate MPI. However, MPI was higher in AS patients than the control group by using TDI. It was because IVRTm measured by TDI was longer for AS patients than that of the control group, CTm was shorter significantly, and PCTm was similar between two groups. This result demonstrates that TDI was superior to conventional methods for diagnosing left ventricular dysfunction in patients with AS. We are unable to explain why only some parameters were changed and the others remained the same. It might be related to the fact that occurrence of diastolic dysfunction requires a long time throughout the course of the disease and different parameters were disturbed at different stages.

TDI has some limitations since it could be affected from rotations and stretching-extension effects of contracted and relaxed heart. Strain rate imaging is a new echocardiographic method by which segmentary tissue deformation degree can be measured and by this way limitations of TDI are disappeared. The present study has the limitation that strain rate imaging was not used. 
examination in a series of 55 patients. Ann Clin Res 1970;2:106-12.

9. Bergfeldt L. HLA-B27-associated cardiac disease. Ann Intern Med 1997;127:621-9.

1. Roldan CA, Chavez J, Wiest PW, et al. Aortic root disease and valve disease associated with ankylosing spondylitis. J Am Coll Cardiol 1998;32:1397-404.

2. Arnason JA, Patel AK, Rahko PS, Sundstrom WR. Transthoracic and transesophageal echocardiographic evaluation of the aortic root and subvalvular structures in ankylosing spondylitis. J Rheumatol 1996;23:120-3.

3. Alves MG, Espirito-Santo J, Queiroz MV, et al. Cardiac alterations in ankylosing spondylitis. Angiology 1988;39:567-71.

4. Brewerton DA, Gibson DG, Goddard DH, et al. The myocardium in ankylosing spondylitis. A clinical, echocardiographic, and histopathological study. Lancet 1987;1: 995-8.

5. O'Neill TW, King G, Graham IM, et al. Echocardiographic abnormalities in ankylosing spondylitis. Ann Rheum Dis 1992; 51:652-4.

6. Calin A. The Dunlop-Dottridge Lecture. Ankylosing spondylitis: defining disease status and the relationship between radiology, metrology, disease activity, function, and outcome. J Rheumatol 1995;22:740-4.

7. Lehtinen K. 76 patients with ankylosing spondylitis seen after 30 years of disease. Scand J Rheumatol 1983;12:5-11.

8. Takkunen J, Vuopala U, Isomäki H. Cardiomyopathy in ankylosing spondylitis. I. Medical history and results of clinical
17. Peters MJ, van der Horst-Bruinsma IE, Dijkmans BA, Nurmohamed MT. Cardiovascular risk profile of patients with spondylarthropathies, particularly ankylosing spondylitis and psoriatic arthritis. Semin Arthritis Rheum 2004;34:585-92.

18. Trambaiolo P, Tonti G, Salustri A, et al. New insights into regional systolic and diastolic left ventricular function using tissue Doppler echocardiography: from qualitative analysis to a quantitative approach. J Am Soc Echocardiogr 2001;14:85-96.

19. Gulati VK, Katz WE, Follansbee WP, Goresan J. Mitral annular descent velocity by tissue Doppler echocardiography as an index of global left ventricular function. Am J Cardiol 1996;77:979-84.

20. Oki T, Tabata T, Mishiro Y, et al. Pulsed tissue Doppler Imaging of left ventricular systolic and diastolic wall motion velocities to evaluate differences between long and short axes in healthy subjects. J Am Soc Echocardiogr 1999;12:308-13.

21. Okan T, Sari I, Akar S, et al. Ventricular diastolic function of ankylosing spondylitis patients by using conventional pulsed wave Doppler, myocardial performance index and tissue Doppler imaging. Echocardiography 2008;25:47-56.

22. Caliskan M, Erdogan D, Gullu H, et al. Impaired coronary microvascular and left ventricular diastolic functions in patients with ankylosing spondylitis. Atherosclerosis 2008;196:306-12. 